\title{
Coupled ion-interface dynamics and ion transfer across the interface of two immiscible liquids
}

\author{
A. A. Kornyshev \\ Institute for Materials \& Processes in Energy Systems, Research Center "Jülich," 52425 Jülich, Germany \\ and Department of Chemistry, Faculty of Physical Sciences, Imperial College of Science, \\ Technology and Medicine, SW72AY London, United Kingdom \\ A. M. Kuznetsov \\ Department of Electrochemistry, University of Ulm, D-89081 Germany and The A. N. Frumkin Institute of \\ Electrochemistry of the Russian Academy of Sciences, 117071 Moscow, Russia \\ M. Urbakha) \\ School of Chemistry, Tel Aviv University, Ramat Aviv, 69978 Tel Aviv, Israel
}

(Received 25 March 2002; accepted 17 July 2002)

\begin{abstract}
When an ion moves across the interface of two immiscible electrolytes it moves together with the ion-induced protrusion of one solvent into the other. For an infinitely slow motion of an ion the height of the protrusion, $h_{\mathrm{eq}}$, is a function of the position of the ion $z$. Due to a finite relaxation time the protrusion may not be able to spontaneously follow the motion of the ion, and this will cause slowing down of the ion transfer. The relaxation of the protrusion involves the movements of many solvent molecules and must be considered on the same footing as the motion along the coordinate of the ion. In this paper we develop a theory of such coupled motion which determines the kinetic laws of the ion transfer across the interface. When the equilibrium electrochemical potential for the ion has no barrier, the process of ion transport is purely diffusional and the effective diffusion coefficient may be evaluated as $D_{\text {eff }}=k_{B} T /\left\{6 \eta\left[r_{i}+(4 / 3)\left(h_{\max } / \Lambda\right)^{2} L\right]\right\}$, where $\eta$ is the average viscosity of the liquids, $r_{i}$ is the Stokes radius of an ion, $L$ and $h_{\max }$ is the lateral size and the maximal height of the protrusion, and $\Lambda$ is the half width of the function $h_{\mathrm{eq}}(z)$, which characterizes equilibrium ion-interface coupling. When there is a barrier, the theory recovers, depending on the height of the barrier, the mechanisms of ion transfer considered by Marcus or Gurevich-KharkatsSchmickler. The effect of the nature of the ion and the solvents in contact is discussed. (c) 2002 American Institute of Physics. [DOI: 10.1063/1.1505862]
\end{abstract}

\section{INTRODUCTION}

Water and oil at ambient conditions do not mix. In equilibrium, a stable interface is formed between them. Although it fluctuates due to thermally excited capillary waves, ${ }^{1,2}$ its identity is preserved at ambient temperatures. Typical examples of organic phases used to create such interfaces are nitrobenzene and 1,2-dichlorethane. Such interfaces have been used for the phase transfer catalysis ${ }^{3}$ and in "soft matter" electrochemistry. ${ }^{4}$

Most of the standard electrolytes are composed of hydrophilic ions that dissolve eagerly in water, rather than in organic liquids. But there are salts of another kind, composed of ions that can dissolve both in water and oil phase, but have higher affinity to oil. Their hydration energies in the oil phase are higher than in water and they prefer the oil phase. Such ions will be conventionally called "hydrophobic" although they dissolve in water too. Examples are the large tetralkylammonium cations and tetraphenylborate anion. ${ }^{5}$

A mixture of salts composed of hydrophilic ions and of salts composed of hydrophobic ions will distribute between the two liquids in contact. Hydrophobic ions will go to the organic phase, while the hydrophilic ions will dissolve in the

\footnotetext{
${ }^{a)}$ Author to whom correspondence should be addressed. Electronic mail: urbakh@post.tau.ac.il
}

aqueous phase. Up to a minor penetration of such salts into the unfriendly liquid, ${ }^{6,7}$ this system forms two "back-toback" electrical double layers and can be electrically polarized.

In addition to these two buffer electrolytes one may add a small amount of salt that contains an ion less tolerant to one of the solvents, whose chemical potential in the bulk of the "unfriendly" solvent is higher, but not very much higher. Moderately polarizing this system, i.e., creating the external field perpendicular to the interface, one can compensate the difference in chemical potentials of that ion in the two phases and study the laws of the ion transfer across the interface. If all other ions have much larger differences between the chemical potentials in the bulk of the two solvents, measuring the electric current across the interface one can characterize the mobility of the given ion.

Electrochemistry of the interface of immisible electrolyte solutions (ITIES) has developed into a mature discipline. Valuable practically, it is considered to be a nifty model system (in particular, biomimetic) where the ion-transport dynamics across the interface can be studied by electrochemical impedance spectroscopy or chronoamperometry. ${ }^{4,5}$ Measuring the ionic current normal to the interface it was found that the effective mobility of ion passage across the interface is much slower than the mobility of the same kind of ion in the 
bulk of each of the liquids in contact. The strength of this effect has "varied in time:" earlier reports claimed the mobility decrease of several orders of magnitude, whereas recent observations for the same systems seem to reveal hardly more than 2 orders of magnitude. ${ }^{8}$ It is not completely clear whether this is related to some changes of experimental conditions or the increase of experimental accuracy. What is obvious, however, is that there is a phenomenon of slowing down the ion mobility at the interface. A challenge for the theory is to explain it, to estimate its value, and to reveal the factors that may affect it.

From the very beginning, the kinetics of ionic transfer was considered as a first-order heterogenous reaction. ${ }^{9}$ To go further it was necessary to specify the reaction mechanism. Shao et al. ${ }^{10,11}$ suggested treating ion transfer as a diffusion flux across the interface. This approach has been elaborated by Kakiuchi, ${ }^{12}$ who adopted a constant field, Goldman approximation, ${ }^{13}$ and got an analytical result for the rate constant as a function of potential drop across the interface. According to this theory, in order to explain experimental data, the ion diffusion coefficient in the interfacial region is to be assumed much smaller than its bulk value in either of the liquids. ${ }^{8}$ A number of authors focused attention on the role of the profile of electrochemical potential of the ion. Schmickler ${ }^{14}$ has pointed out that the combination of monotonic profiles of the chemical potential and the electrical potential of the polarized interface may give rise to a potential well and a barrier in the electrochemical potential. Therefore the ion transfer was expected to be an activation process; Schmickler has studied the potential dependence of the barrier height and of the transfer coefficient. Some speculations on the possible mechanisms for the maximum in the chemical potential were earlier reported. ${ }^{15}$ Indenbom ${ }^{16}$ suggested that the ion-induced corrugation of the interface should remove the maximum in the chemical potential. The existence of the ion-induced corrugation of the interface has been demonstrated in molecular dynamics simulations by Benjamin. ${ }^{17}$ No maximum in the chemical potential was found. ${ }^{18}$

Recently, Marcus published a most stimulating essay ${ }^{19}$ with a detailed discussion of the experimental and theoretical aspects of the problem. We refer the reader to that paper for a detailed review of experimental situation and various phenomenological and molecular dynamic approaches to this problem. Stimulated by some results in the computer simulations of Benjamin, ${ }^{17}$ he has suggested and explored an original idea that ion transport across the interface is assisted by a favorable deformation of the surface, i.e., a protrusion of one phase into the other. He considered such a protrusion to be a precursor of the ion motion across the interface and the fluctuation-induced inversion of the protrusion to be the factor facilitating the interfacial transfer. According to Marcus estimates, the probability of the fluctuation essentially determines the ionic mobility across the interface.

Marcus has stressed that not only is the ion motion affected by such fluctuations, but the ion may in turn influence the shape of surface. However, this idea was not explored in his work. He has drawn attention to the fact that fluctuating protrusions can facilitate the ion transfer across the interface, but the direction of the protrusion was not important in his
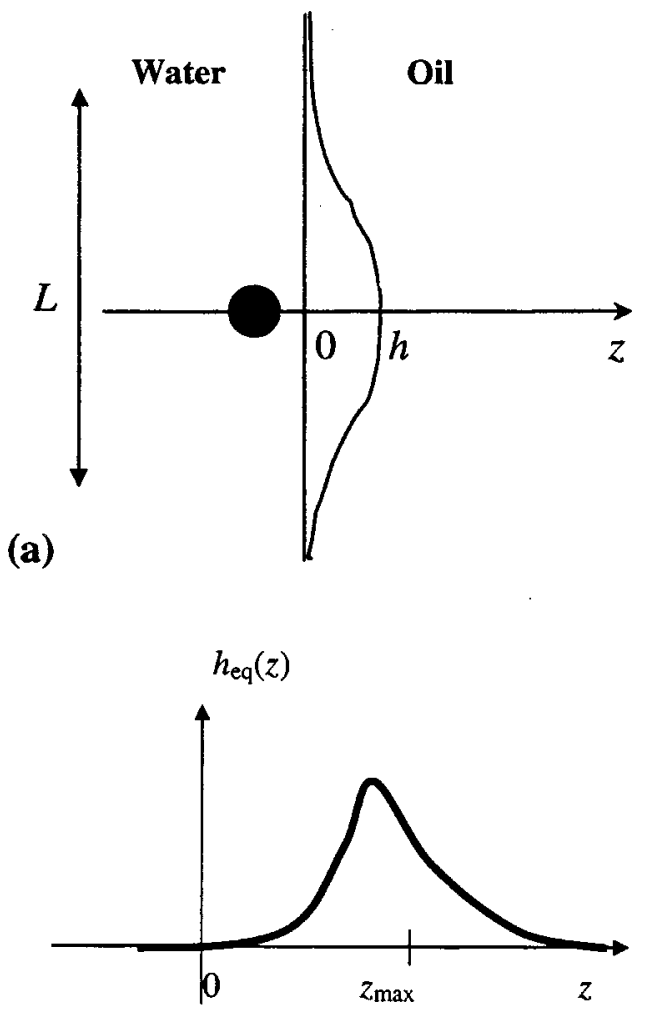

(b)

FIG. 1. (a) Protrusion at a water-oil interface in the presence of a hydrophilic ion. (b) The height of the equilibrium protrusion as a function of the distance of the hydrophilic ion from the interface.

estimates, and there was no explicit coupling between the ion motion and the protrusion coordinate. As will be shown in the present paper, such feedback may have a dramatic effect on the kinetics of ion transport across the interface.

Our approach rests on the following considerations. Let us take a water/oil system which in an unperturbed state has a flat interface at $z=0$, with the oil half space at $z>0$, and water half space at $z<0$. Consider a hydrophilic ion, which feels itself comfortable in water but is driven toward the oil phase by an external field. Consider the ion moving infinitely slowly toward the interface. On the way to the interface it will tend to keep water around itself. This will create a bump, i.e., water protrusion into the oil, in front of the ion [Fig. 1(a)]. Interface tension will, however, not favor deep protrusions of the water phase into the oil. Thus, after a certain penetration depth, the ion strips water off. This can be visualized as if the ion skewers the bump surface. After the ion crosses the surface, the latter "returns back" to a flattened state. Of course, if the ion attracts water stronger than water is repelled from the oil, the ion will keep its first hydration shell even in the bulk of the oil phase. Then the ion traverses the surface bump together with its first hydration shell. If the hydrophilic ion moves from oil to water, it induces water protrusion into the oil. After the ion crosses the protrusion surface, the latter relaxes to the flat state.

Let us denote the height of the bump by $h_{\text {eq }}$, or more precisely the position of the maximum height of the water protrusion in front of the ion, for a given position of the ion 

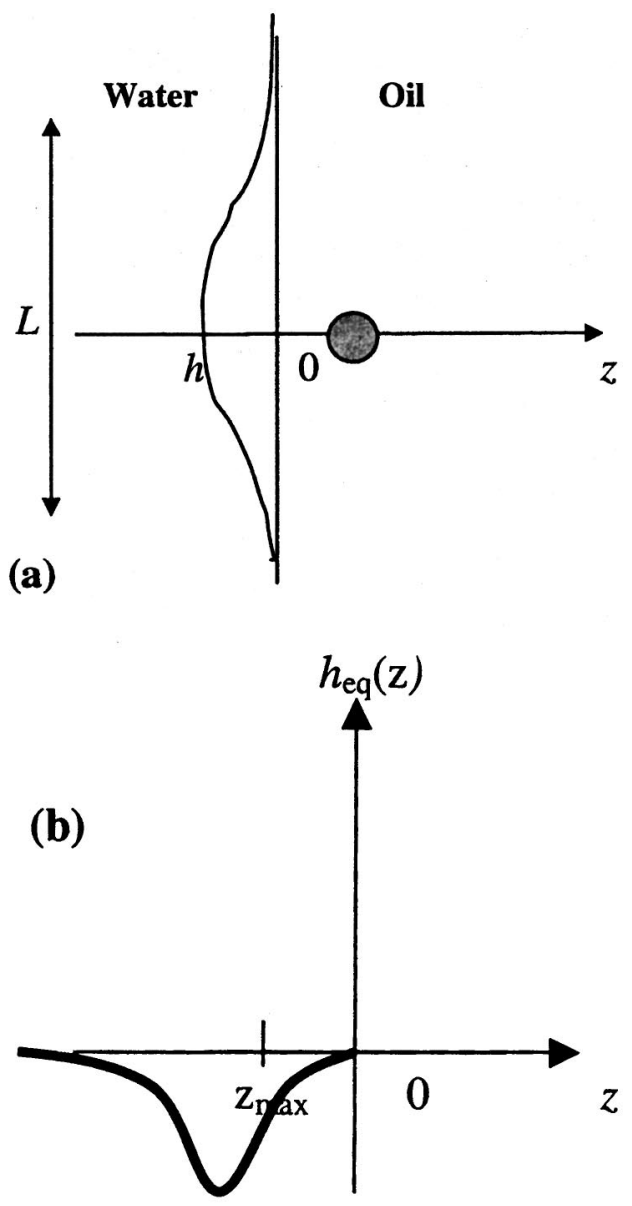

FIG. 2. (a) Protrusion at a water-oil interface in the presence of a hydrophobic ion. (b) The height of the equilibrium protrusion as a function of the distance of the hydrophobic ion from the interface.

"fixed" at point $z$. This means that the equilibrium profile of $h_{\mathrm{eq}}(z)$ for a hydrophilic ion is positively defined and has a maximum at $z>0$ [Fig. 1(b)]. Note that the mere electrostatic effect of the ion charge will cause such an effect, because the dielectric polarizability of water is much higher than that of oil. Both short range hydrophilicity and image forces near the interface will work in one direction, moving the maximum of $h_{\mathrm{eq}}(z)$ toward more positive values.

The situation with a hydrophobic ion (Fig. 2) is slightly more complicated. The charge of the ion will tend to make $h_{\text {eq }}(z)$ positive with a maximum at $z>0$ but the short-range interaction (dominating at short distances because the ion in spite of its charge prefers to dissolve in oil, rather than in water) will produce an opposite effect. Moving in oil toward water or in water toward oil such ion induces a protrusion of oil into the aqueous phase. The value of $h_{\mathrm{eq}}$ will, presumably, be negative with the maximum at negative $z$ but, due to a competition between the charge effect and the short range effect, $\left|h_{\mathrm{eq}}(z)\right|$ will likely be smaller than for a hydrophilic ion.

In reality the ion does not move infinitely slowly and the liquid-liquid surface has a finite relaxation time. Furthermore, the surface undergoes spontaneous dynamic corrugations, due to capillary waves. In spite of that, the ion and the surface will try to move in harmony with each other. How does that influence the kinetics of ion transfer across the interface?
Imagine that the ion "flies" across the interface with such speed that the surface has no time to react to the momentary position of the ion. The barrier for the ion passage would then be higher than in the case when it had moved slower. That tells us that the ion transfer process is determined by the motions in the space of two reaction coordinates: the coordinate of the ion $z$ and a "collective" coordinate $h$, which characterizes the protrusion. The system chooses an optimum pathway in the space of these two variables. In this paper we will treat both coordinates on the same footing, taking into account the coupling between them through the $h_{\mathrm{eq}}(z)$. We will map the theory on the stochastic two-dimensional Kramers problem. ${ }^{20}$

Note that role of stochastic dynamics in the context of ion transfer across the liquid-liquid interface has been discussed by Gurevich and Kharkatz, ${ }^{21}$ Kharkatz and Kuznetsov, ${ }^{22}$ and Schmickler. ${ }^{14}$ However only onedimensional motion along the coordinate of the ion has been considered. Therefore, the effect of protrusion fluctuations was beyond consideration. The necessity to include additional solvation coordinates has been stressed in the context of ion transfer reaction at electrodes. ${ }^{23-25}$

In this paper, the closed form solutions of this problem are obtained in two limiting cases: when (i) the motion of ions is faster than the relaxation of the bump (the case of conventional ions) and (ii) the motion of ions is slower than the motion of the degrees of freedom of the bump (abnormally heavy ions). In the case when there is no maximum in the electrochemical potential of an ion, the results suggest a transparent interpolation between the two limits, offering a unified description for an effective diffusion coefficient of the ion in the interfacial region. The case of a maximum in the electrochemical potential, caused by a competition of the chemical and electric potentials ${ }^{14}$ and/or nonmonotonic chemical potential, is considered separately.

\section{EQUILIBRIUM ION-INDUCED PROTRUSION OF THE INTERFACES}

Let us discuss the physics of the bump formation and consider a simplified example, which rationalizes the $h_{\mathrm{eq}}(z)$ profile. Consider an ion, which is infinitely slowly driven from the interior of water into oil along the coordinate $z$, perpendicular to the interface, so that the interface can adjust its equilibrium shape subject to different positions of the ion. When the ion is far from the interface (either in oil or in water), the equilibrium shape of the interface is flat. As it has been explained in Sec. I, approaching the interface from the aqueous side induces a protrusion (bump) (Fig. 1), because the ion, unless it is highly hydrophobic, likes to be surrounded by the liquid of stronger polarity. Moving the ion further closer to the interface, we increase the height of the bump, $h_{\text {eq }}$. The surface tension of the interface prevents, however, the unlimited increase of the height. At a certain curvature of the bump, determined by the compromise between the repulsion of the interface from the ion and the force caused by the increase of the interfacial area, the ion crosses the interface. With the ion moving further from the interface into the oil, the height of the bump decreases, flattening in the end. Thus $h_{\mathrm{eq}}(z)$ is a curve with a maximum 
and decaying wings on the two sides of the surface, at $z \rightarrow \pm \infty$. It remains to learn where the position of the maximum $z_{\max }$ lies, how high the maximum $h_{\max }$ is, and how large the width $L$ of the function $h_{\mathrm{eq}}(z)$ is.

In order to get a feeling about the shape of $h_{\mathrm{eq}}(z)$, we first consider the ion just as a charge, interacting with the interface via image forces of classical electrostatics. ${ }^{26} \mathrm{Mo}-$ tion of the charge in the aqueous phase will repel the interface electrostatically. The shape of the bump induced by such charge has been studied, ${ }^{27,28}$ but we present here a more dubious estimate, whose roughness is a price for its transparency.

We first estimate the image force that pushes the interface away from the ion of charge $q$ in water as

$$
\frac{L_{B}^{w} \nu}{4\left\{\left(h_{\mathrm{eq}}-z\right)^{2}+d^{2}\right\}} k_{B} T .
$$

Here, $\nu=\left(\epsilon_{w}-\epsilon_{o}\right) /\left(\epsilon_{w}+\epsilon_{o}\right)$, where $\epsilon_{w}$ and $\epsilon_{o}$ are the dielectric constants of water and oil, respectively, $L_{B}^{w}$ $=q^{2} / \epsilon_{w} k_{B} T$ is the Bjerrum length, $T$ is the temperature, $k_{B}$ is the Boltzmann constant, and $d$ is the cutoff length. The latter is on the order of the radius of the ion or "molecular thickness" of the interface and without it the force would have diverged at $z=h_{\mathrm{eq}}$. The force, which prevents the protrusion from growing, is caused by the surface tension and it may be estimated as $\gamma \pi h_{\mathrm{eq}} / \sqrt{1+\left(h_{\mathrm{eq}} / L\right)^{2}}$, where $L$ is the half width of the bump. In fact, the $h_{\mathrm{eq}} / L$ correction has a small effect on the result. Since we did not take into account the curvature of the bump in the image force term, we will further neglect the $h_{\mathrm{eq}} / L$ correction.

When the ion has crossed the interface and is already in oil we may write the same balance of forces, but with $L_{B}^{w}$ replaced by $L_{B}^{o}$.

Equalizing the two forces we obtain

$$
\begin{aligned}
& \frac{\nu}{8 \pi} \frac{L_{B}^{w}}{\left(h_{\mathrm{eq}}-z\right)^{2}+d^{2}}=p h_{\mathrm{eq}}, \quad z<h_{\mathrm{eq}}, \\
& \frac{\nu}{8 \pi} \frac{L_{B}^{o}}{\left(h_{\mathrm{eq}}-z\right)^{2}+d^{2}}=p h_{\mathrm{eq}}, \quad z>h_{\mathrm{eq}},
\end{aligned}
$$

where $p=\gamma / 2 k_{B} T$. It is easy to resolve these equations in terms of $z\left(h_{\mathrm{eq}}\right)$. Hence,

$$
\begin{aligned}
& z=h_{\mathrm{eq}}+\sqrt{\frac{\xi L_{B}^{o}}{h_{\mathrm{eq}}}-d^{2}} \quad \text { (in oil), } \\
& z=h_{\mathrm{eq}}-\sqrt{\frac{\xi L_{B}^{w}}{h_{\mathrm{eq}}}-d^{2}} \quad \text { (in water), }
\end{aligned}
$$

where

$$
\xi=\frac{\nu}{8 \pi p} .
$$

The upper and lower equations fix different limiting values of $h_{\text {eq }}: h_{\max }=\left(\xi / d^{2}\right) L_{B}^{o}$ and $h_{\max }=\left(\xi / d^{2}\right) L_{B}^{w}$, where $L_{B}^{o}$ can be one order of magnitude larger than $L_{B}^{w}(\approx 7 \AA$ for a monovalent ion).

Note, that Eq. (3) suggests that $z\left(h_{\mathrm{eq}}\right)$ is a two valued function, i.e., when the ion moves in the oil toward the in-

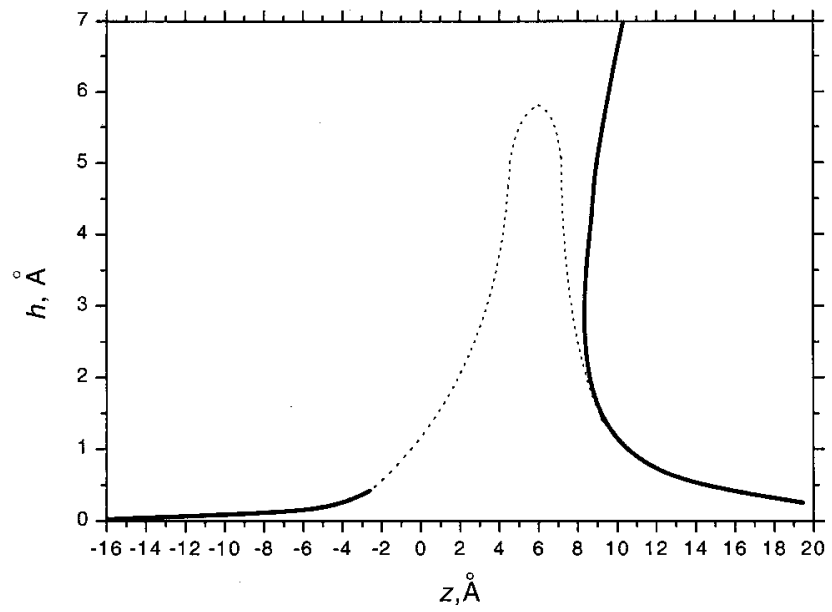

FIG. 3. The height of the equilibrium protrusion as a function of the distance of a point charge from the interface of two dielectric media with dielectric constants 80 and 4.75. Solid lines display the results of calculations according to Eqs. (3) and (4), dashed line is an extrapolation into the interfacial region.

terface the charge-induced water protrusion into oil can increase jumpwise at a certain positive $z$, whereas if the ion moves away from the interface the protrusion will collapse jumpwise at some other $z$. All in all, $h_{\mathrm{eq}}(z)$ will exhibit hysteresis. In reality, this will hardly be true, because at short distances due to the effects of spatial dispersion ${ }^{29,30}$ and the smearing of the interface ${ }^{31} \xi$ vanishes in the interfacial region, and the two branches must merge. Also, if we speak about real ions, the short-range forces will interfere in the force balance equation. If there are oscillating contributions to the forces, due to molecular packing, the resulting $h_{\mathrm{eq}}(z)$ plot may be wavy "at molecular resolution." However, the envelope should have one distinct maximum. A range of $z$ around this maximum separates the two branches given by Eqs. (3) and (4). At the wings the latter give

$$
\begin{aligned}
& h_{\mathrm{eq}}=\frac{\xi L_{B}^{o}}{z^{2}} \quad \text { (in oil), } \\
& h_{\mathrm{eq}}=\frac{\xi L_{B}^{w}}{z^{2}} \quad \text { (in water). }
\end{aligned}
$$

This plot $h_{\mathrm{eq}}(z)$ is shown in Fig. 3. The intermediate region is drawn schematically. The maximum of the schematic curve must lie on a bisector, where $h_{\mathrm{eq}}=z$. A spectacular feature is the asymmetry of $h_{\mathrm{eq}}(z)$ around $z=0$. It causes positive values of the position of the maximum $z_{\max }$. The latter will be shifted even more positively for the case of an additional short-range hydrophilicity of the ion. For the ions that reveal a competing hydrophobicity, as in the case of large tetralkylammonium ions, the position of the maximum would be shifted back, toward $z=0$ or even negative values, as discussed in Sec. I. These simple speculations are in line with the existing computer simulations. ${ }^{17}$

For a given combination of the liquids in contact, the shape of $h_{\mathrm{eq}}(z)$, including the position and the height and the width of the maximum, is a feature of hydration of the ion at the interface, but the nature of the liquids also influences this 
shape through the surface tension. The higher the surface tension, the lower the absolute value of the maximum (c.f. the inversely proportional dependence of the height of the branches to $p$ through $\xi$ ). The electrostatic effect on $h_{\mathrm{eq}}(z)$ is sensitive to the polarity of the liquids: The stronger the difference in polarity, the more positive is the position of $h_{\max }$.

\section{COUPLED DYNAMICS OF ION AND INTERFACIAL PROTRUSION}

Under an assumption of the diffusional character of the motion of the ion and of local deformations of the interface, the Langevin equations ${ }^{20}$ for the coupled ion and interfacial protrusion dynamics read

$$
\begin{aligned}
& \eta_{z} \frac{d z}{d t}+\frac{\partial V(z, h)}{\partial z}=f_{z}(t), \\
& \eta_{h} \frac{d h}{d t}+\frac{\partial V(z, h)}{\partial h}=f_{h}(t) .
\end{aligned}
$$

Here $z$ is the distance of the ion from the flat interface, $h$ is a momentary height of the protrusion at the interface [as in Figs. 1(a) and 2(a)], and $\eta_{z}$ and $\eta_{h}$ are the dissipation constants for the ion and surface motions, respectively. The effect of thermal motion is described by $\delta$-correlated random forces, ${ }^{20} f_{z}$ and $f_{h}$

$$
\begin{aligned}
& \left\langle f_{z}(t) f_{z}\left(t^{\prime}\right)\right\rangle=2 k_{B} T \eta_{z} \delta\left(t-t^{\prime}\right), \\
& \left\langle f_{h}(t) f_{h}\left(t^{\prime}\right)\right\rangle=2 k_{B} T \eta_{h} \delta\left(t-t^{\prime}\right),
\end{aligned}
$$

where $\delta\left(t-t^{\prime}\right)$ is Dirac delta function. We note that the parameters $D_{z}=k_{B} T / \eta_{z}$ and $D_{h}=k_{B} T / \eta_{h}$ represent, respectively, the diffusion coefficients of the ion and of the fluctuating interface.

The equations of motion (8) and (9) are coupled through the potential $V(z, h)$. In accordance with a capillary wave theory $^{32}$ we assume that the excess surface energy due to the fluctuations is proportional to the square of the deviation of the interface position $h$ from the equilibrium value $h_{\mathrm{eq}}(z)$. The potential $V(z, h)$ also includes a variation of the equilibrium electrochemical potential $\mu(z)$ of the ion across the interface, the one that corresponds to the infinitely slow motion of the ion. Then $V(z, h)$ can be written in the form

$$
V(z, h)=\mu(z)+\frac{1}{2} K\left[h-h_{\mathrm{eq}}(z)\right]^{2},
$$

where $h_{\mathrm{eq}}(z)$ is the equilibrium value of the height of the protrusion for an infinitely slow variation of $z$ (cf. Sec. II).

The assumptions, underlying Eqs. (8)-(9) are as follows:

(i) both the ion and the interface motion are considered being overdamped,

(ii) the protrusions are characterized by its height in the maximum only, and

(iii) variation of ion diffusion coefficient across the interface is neglected.

The first assumption is well justified for the ionic motion and short wavelength fluctuations of the interface in a viscous liquid. It is valid in this problem, because only the fluctuations with short wavelengths (on the order of the protrusion-bump width) influence the interfacial ion transfer. The second simplification means that we consider interfacial protrusions with a given lateral scale (wave vector $q_{0}$ ) only, assuming that they give the major contribution to the ioninterface interaction. Finally, the ion diffusion coefficient varies monotonically between the corresponding values in the contacting liquids, ${ }^{33}$ and thus it cannot vary more than twice, because that is the typical maximum ratio of the bulk viscosities of the two solvents.

In the absence of ion-interface coupling, $h_{\mathrm{eq}}(z)=0$. Equation (8) then reduces to the Nernst-PlanckSmoluchowski equation for ionic transfer across the interface, ${ }^{12}$ whereas Eq. (9) coincides with the equation for overdamped capillary fluctuations. ${ }^{31}$

Parameters $\eta_{h}$ and $K$, which enter Eqs. (8)-(10), can be estimated on the basis of the capillary wave theory and expressed through the interfacial tension $\gamma$ and viscosities of the contacting media $\eta_{1}$ and $\eta_{2}$

$$
K=\gamma, \quad \eta_{h}=2\left(\eta_{1}+\eta_{2}\right) / q_{0},
$$

where $q_{0}=2 \pi / L$ and $L$ is a characteristic lateral (along the interface) size of the protrusion.

The mechanism of ion transfer depends on both equilibrium and dynamic characteristics of the system, namely on the form of $\mu(z)$ profile in the interfacial region and on the ratio of diffusion coefficients of the ion and of the interface. As we have already noted in Sec. I, both monotonic behavior of $\mu(z)$ and that containing maximum at the interface have been considered to describe ionic transfer across ITIES. ${ }^{12,14-16,18,19}$ Below we will consider separately the cases of monotonic and nonmonotonic $\mu(z)$ because, as it appears, the presence of a minimum and maximum in $\mu(z)$ has a dramatic effect on the ion transfer mechanism. Equation (11) for the potential $V(z, h)$ suggests that even for the case of monotonic behavior of $\mu(z)$, a deviation of the momentary shape of the interface from the equilibrium, $h_{\mathrm{eq}}(z)$, leads to a nonmonotonic shape of the potential acting on the ion.

\section{FOKKER-PLANCK DESCRIPTION OF THE IONIC TRANSFER ACROSS ITIES}

In order to calculate the ionic current it is convenient to transform the coupled Langevin equations (8) and (9) to a Fokker-Planck equation $^{20}$ for the probability $w(z, h, t)$ to find at a time moment $t$ the ion at position $z$ and the protrusion having height $h$. Hereafter $w(z, h, t)$ will be called distribution function. This equation reads

$$
\frac{\partial w(z, h, t)}{\partial t}=-\left[\frac{\partial J_{z}(z, h)}{\partial z}+\frac{\partial J_{h}(z, h)}{\partial h}\right] w(z, h, t),
$$

where $J_{z}(z, h, t)$ and $J_{h}(z, h, t)$ are the flux operators in $z$ and $h$ directions

$$
\begin{aligned}
& J_{z}(z, h)=-\frac{1}{\eta_{z}}\left(k_{B} T \frac{\partial}{\partial z}+\frac{\partial V(z, h)}{\partial z}\right), \\
& J_{h}(z, h)=-\frac{1}{\eta_{h}}\left(k_{B} T \frac{\partial}{\partial h}+\frac{\partial V(z, h)}{\partial h}\right) .
\end{aligned}
$$


The ionic concentration $c(z, t)$ is expressed through the distribution function as

$$
c(z, t)=\int_{-\infty}^{+\infty} d h w(z, h, t) .
$$

The solution of Eq. (13) should obey the following boundary conditions:

$$
w(z, h \rightarrow \pm \infty, t) \rightarrow 0,
$$

and

$$
\int_{-\infty}^{+\infty} d h w\left(z_{i}, h, t\right)=c\left(z_{i}\right)
$$

and

$$
\int_{-\infty}^{+\infty} d h w\left(z_{f}, h, t\right)=c\left(z_{f}\right)=0
$$

where $z_{i}$ and $z_{f}$ are the points of entrance of the ion into the interfacial region in phase 1 and the point of departure from it in phase 2 , and $c\left(z_{i}\right)$ and $c\left(z_{f}\right)$ are the concentrations of the transferable ion at $z=z_{i}$ and $z=z_{f}$. Hereafter we assume that the final ionic concentration $c\left(z_{f}\right)$ equals zero, and calculate the rate constant corresponding to the transfer from phase 1 to phase 2 (forward reaction). ${ }^{34}$

Exact analytical solutions of Eq. (13) can be obtained in two limiting cases: (i) the motion of the interfacial protrusion is faster than the motion of ions, $D_{h} \gg D_{z}$, and (ii) the motion of the protrusion is slower than the motion of ions, $D_{h}$ $\ll D_{z}$. The motion of the interfacial protrusion involves a larger number of molecules than the motion of the ion and it is logical therefore to expect that $D_{h}$ is smaller than $D_{z}$. Thus the limiting case (ii) should be most suitable for a description of the ionic transport across ITIES.

As we have already mentioned above the mechanism of the ionic transfer depends not only on the kinetic coefficients $D_{z}$ and $D_{h}$, but also on the shape of equilibrium electrochemical potential $\mu(z)$. In order to demonstrate this point in Fig. 4 we present the potential energy surfaces of $V(z, h)$ for the cases of $\mu(z)=$ constant [Fig. 4(a)] and $\mu(z)$ having a barrier in the interfacial region [Fig. 4(b)]. One can see that in the first case the ion moves from the initial point $z=z_{i}$ to the final one $z=z_{f}$ performing pure diffusion motion inside the potential groove. However, the description of this motion should differ essentially from the traditional model of the diffusion transfer of the ion across the interface. ${ }^{10,12}$ The latter does not include an interaction with the fluctuations of the interface and as a result considers the diffusion of ion along the $z$ coordinate only. Figure 4 shows that the coupled system, which includes the ion and the protrusion, performs a two-dimensional diffusion in both $z$ and $h$ directions. The ionic current should be determined by the slowest of these processes. In the second "barrier" case the ion has to overcome the barrier either in the $z$ or $h$ direction and one can expect an activation mechanism of the ionic transfer.

In the limiting cases discussed above the solution of the problem can be found following the procedure of adiabatic
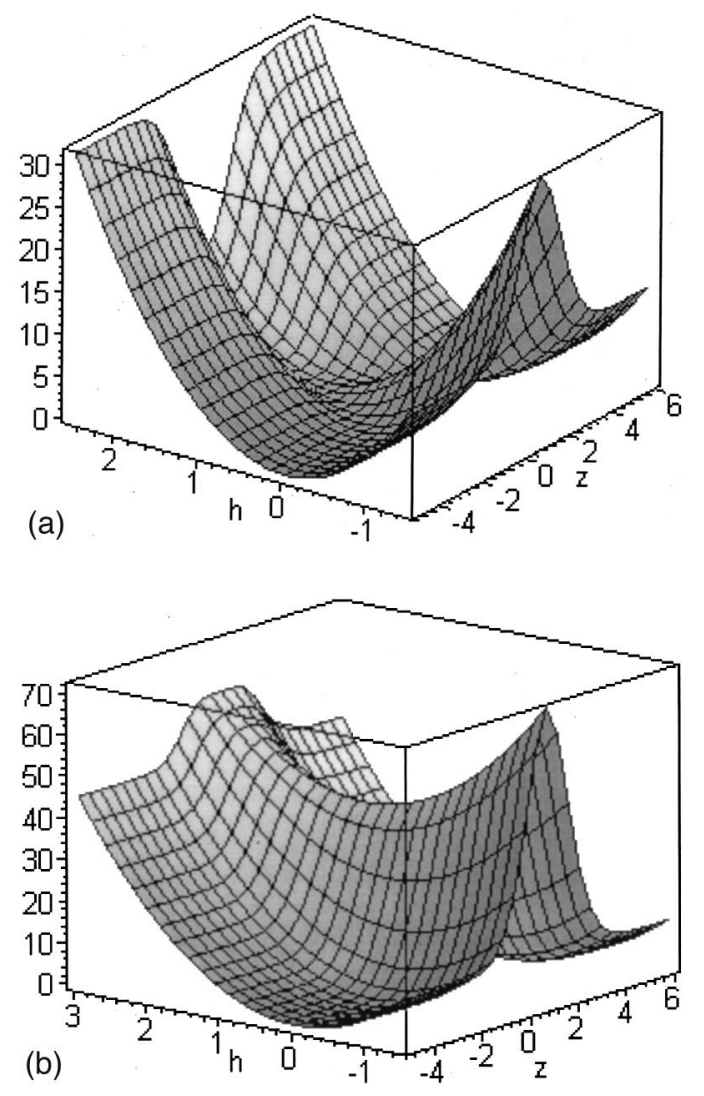

FIG. 4. Surfaces of the potential energy $V(z, h)$ : (a) constant electrochemical potential, and (b) electrochemical potential having a barrier in the interfacial region.

elimination of the fast variable, ${ }^{20}$ which is outlined in Appendix A. In the following sections we discuss the analytical results obtained for different limiting cases.

\section{ION TRANSFER FOR A MONOTONIC VARIATION OF THE ELECTROCHEMICAL POTENTIAL}

\section{A. Slow motion of the ion}

For tutorial purposes we will start with the less actual limiting case $D_{h} \gg D_{z}$, which is of practical interest only for abnormally large ions. In this case, the interfacial dynamics should give only a small correction to the traditional NerstPlanck equation ${ }^{12}$ for the ionic current and it is easy to obtain it. The slowness of motion along the $z$ coordinate results in the equilibrium distribution over $h$ being established for every position of the ion. Then averaging the Fokker-Planck equation over fluctuations of the interface and taking into account the terms of the zero and the first order in the small parameter $\delta=D_{z} / D_{h}$, we arrive at the following equation on the ionic concentration $c(z)$ (see Appendix B):

$$
\begin{aligned}
\frac{\partial c(z, t)}{\partial t}= & D_{z} \frac{\partial}{\partial z}\left[\left(1-\frac{D_{z}}{D_{h}}\left(\frac{d h_{\mathrm{eq}}(z)}{d z}\right)^{2}\right) \frac{\partial c(z, t)}{\partial z}\right. \\
& \left.+\frac{c(z, t)}{k_{B} T} \frac{d \mu(z)}{d z}\right] .
\end{aligned}
$$

Equation (19) has a form of the Nernst-Planck equation but with an effective diffusion coefficient 


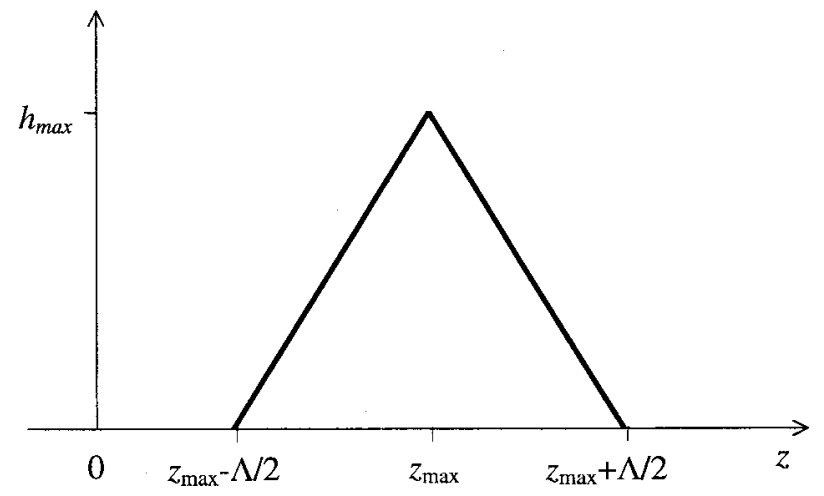

FIG. 5. Model of triangular shape of the function $h_{\mathrm{eq}}(z)$.

$$
D_{\text {eff }}(z)=D_{z}\left(1-\frac{D_{z}}{D_{h}}\left(\frac{d h_{\mathrm{eq}}(z)}{d z}\right)^{2}\right),
$$

which depends on the distance from the interface. In this limit the effect of fluctuating interfacial protrusions on the ion transfer reduces to a distant dependent decrease of the diffusion coefficient of the ion. It should be noted that the protrusion-induced correction to $D_{z}$ is determined by the ratio of the ion and interface diffusion coefficients $D_{z} / D_{h}$, which is a small parameter in this case.

Usually ion transfer reactions across an ITIES are described in terms of rate constant $k_{\text {rate }}$ which is defined by the equation

$$
J=k_{\text {rate }} c\left(z_{i}\right),
$$

where $J$ is the ionic current. Under a steady-state condition, $\partial c / \partial t=0$, Eq. (19) gives the following expression for the rate constant:

$$
\begin{aligned}
k_{\mathrm{rate}}= & D_{z} / \int_{z_{i}}^{z_{f}} d z\left\{\exp \left[\left(\mu(z)-\mu\left(z_{i}\right)\right) / k_{B} T\right]\right. \\
& \times\left[1+\frac{D_{z}}{D_{h}}\left(\left(\frac{d h_{\mathrm{eq}}(z)}{d z}\right)^{2}\right.\right. \\
& \left.\left.\left.+\frac{1}{k_{B} T} \int_{z_{i}}^{z} d z^{\prime} \frac{d \mu\left(z^{\prime}\right)}{d z}\left(\frac{d h_{\mathrm{eq}}\left(z^{\prime}\right)}{d z}\right)^{2}\right)\right]\right\} .
\end{aligned}
$$

At the zero driving force, $\mu\left(z_{i}\right)=\mu\left(z_{f}\right)$, this equation can be simplified and it reads as

$$
k_{\text {rate }}=D_{z} /\left[z_{f}-z_{i}+\left(D_{z} / D_{h}\right) \int_{z_{i}}^{z_{f}} d z\left(\frac{d h_{\mathrm{eq}}(z)}{d z}\right)^{2}\right] \text {. }
$$

As it was expected, the finite rate of relaxation of the interface (finite $D_{h}$ ) reduces the rate constant, as compared to the "traditional" result ${ }^{12}$

$$
k_{\text {rate }}^{\text {dif }}=D_{z} /\left[z_{f}-z_{i}\right] .
$$

However, for $D_{z} \ll D_{h}$ this effect is small. For $h_{\mathrm{eq}}(z)=0$, Eq. (22) coincides with the traditional result Eq. (23).

In order to show explicitly the dependence of the rate constant on the parameters of ion-interface interaction we use a model, the triangular shape of $h_{\mathrm{eq}}(z)$ shown in Fig. 5. Then Eq. (22) transforms to

$$
k_{\text {rate }}=D_{z} /\left[z_{f}-z_{i}+2\left(D_{z} / D_{h}\right) h_{\max }^{2} / \Lambda\right],
$$

where $h_{\max }$ is the maximum height of the protrusion and $\Lambda$ is a half width of the triangle. $\Lambda$ has a meaning of a characteristic distance at which the ionic pressure starts to deform the interface.

\section{B. Fast motion of the ion}

Now consider the most realistic situation when local fluctuations of the interface are slower than the motion of the ion, namely $D_{h}<D_{z}$. In this limit the influence of nonequilibrium interface adjustment manifests itself in the most striking manner. Here the two-dimensional diffusion of the coupled ion-protrusion motion [see Fig. 4(a)] is controlled by the slow $h$ coordinate and the Boltzmann equilibrium over $z$ establishes at every value of $h$. In contrast to the previous case, here one cannot derive an effective equation for the ionic concentration by averaging over the stationary distribution of the $h$ coordinate, because $h$ is now the slow variable. The method of adiabatic elimination of fast variables here requires solving Eq. (13) for the ionic coordinate $z$, keeping the protrusion height fixed, and then deriving an equation of motion for $h$ variable (see Appendix C). For the zero driving force this procedure gives the following expression for the steady state rate constant:

$$
k_{\text {rate }}=\frac{D_{h}}{z_{f}-z_{i}}\left(\frac{\Lambda}{h_{\max }}\right)^{2} \text {. }
$$

In deriving Eq. (25) we assumed that $h_{\mathrm{eq}}(z)$ has the triangular shape and kept only the leading term in the parameter $D_{h} / D_{z}$.

Equation (25) shows that for slow fluctuations of the interface the rate constant is determined by the diffusion coefficient of the interface rather than by the diffusion coefficient of the ion, and this slows down the ion transfer. The nature of the ion-interface interaction (see Sec. II) enters the rate constant through the shape of the functions $h_{\mathrm{eq}}(z)$.

In order to demonstrate a difference between our Eq. (25) for $k_{\text {rate }}$ and the traditional Eq. (23) we estimate the ratio $k_{\text {rate }} / k_{\text {rate }}^{\text {dif }}$ using relationships between the dissipation constants for the ion and the interface, $\eta_{z}$ and $\eta_{h}$, and liquid viscosities $\eta_{1,2}$. Taking into account Eq. (12) and using the Stokes equation $\eta_{z}=6 \pi \eta_{1,2} r_{i}$, where $r_{i}$ is the Stokes radius of the ion, we get

$$
\frac{k_{\text {rate }}}{k_{\text {rate }}^{\text {dif }}} \simeq \frac{3 r_{i}}{4 L}\left(\frac{\Lambda}{h_{\max }}\right)^{2} \text {. }
$$

Here we assumed that $\eta_{1} \approx \eta_{2}$. Equation (26) demonstrates that the experimentally observed rate constant can be much smaller than $k_{\text {rate }}^{\text {dif }}$ since the lateral size $L$ of the protrusion is much larger than the ionic radius $r_{i}$. This conclusion of course holds as far as $\Lambda$ is not much greater than $h_{\text {max }}$, which is, however, hard to expect.

So far we have discussed the case of zero driving force. An analytical solution for $k_{\text {rate }}$ can be also obtained for the linear profile of $\mu(z)$

$$
\mu(z)=\mu\left(z_{i}\right)+\left[\mu\left(z_{f}\right)-\mu\left(z_{i}\right)\right]\left(z-z_{i}\right) /\left(z_{f}-z_{i}\right) .
$$


In this case the solution of Eqs. (13)-(18) gives

$$
k_{\text {rate }}=\frac{D_{h}}{z_{f}-z_{i}}\left(\frac{\Lambda}{h_{\max }}\right)^{2} \frac{u \exp (-u)}{\sinh u},
$$

where

$$
u=\left(\mu\left(z_{f}\right)-\mu\left(z_{f}\right)\right) / 2 k_{B} T .
$$

Expression (28) has the same potential dependence as the traditional result obtained from the solution of the NernstPlanck-Smoluchowskii equation for the linear variation of the electrochemical potential, ${ }^{12}$ but the pre-exponential factor is entirely different.

\section{The general case of an arbitrary ratio between the characteristic velocities of the ion and protrusion motions}

The solutions [Eqs. (24) and (25)] which were found for the rate constants in two opposite limiting cases, $D_{h} \gg D_{z}$ and $D_{h}>D_{z}$, respectively, match naturally in a unified formula for the effective diffusion coefficient,

$$
D_{\mathrm{eff}}=\frac{1}{\frac{1}{D_{z}}+\frac{1}{\left[\Lambda / h_{\mathrm{max}}\right]^{2} D_{h}}} .
$$

This formula reflects the essentially consecutive motion of the ion-protrusion couple along the $z, h$ coordinates, where the coefficient in front of $D_{h}$ takes into account the point where the motion along the $h$ coordinate converts into the motion along the $z$ coordinate.

In terms of the effective radii this result reads

$$
D_{\text {eff }}=\frac{k_{B} T}{6 \eta r_{\text {eff }}}, \quad r_{\text {eff }}=r_{i}+\frac{4}{3}\left[\frac{h_{\text {max }}}{\Lambda}\right]^{2} L,
$$

where we have put for simplicity $\eta \approx \eta_{1} \approx \eta_{2}$. This simple formula shows the renormalization of the Stokes radii due to the ion-protrusion coupling.

\section{FLUCTUATIONALLY ASSISTED ION HOPPING OVER A POTENTIAL BARRIER}

The physical picture of the transition of the ion across the interface is quite different if $\mu(z)$ involves a barrier in the interfacial region (see Fig. 6). The process is then of activation hopping character. Its parameters depend on the relationship between the diffusion coefficients for the ion and for the interface fluctuation. For the sake of simplicity of presentation it is assumed below that there is a potential well just before the barrier.

In the situation where the motion of the interface is much faster than the motion of the ion, the former follows the ion motion and the transition reduces to the ion transfer over the equilibrium barrier $\mu(z)$. In this limiting case the result for the rate constant reduces to that discussed in the literature. ${ }^{14,21,22}$

More interesting is the opposite limit where the ion is faster than the fluctuations of the interface. In this case we can speak about the probability of transition (per unit time) of the ion over the barrier formed by the potential $V(z, h)$ at any instant $h$ value $W_{i \rightarrow f}(h)$ (see Appendix D). The potential

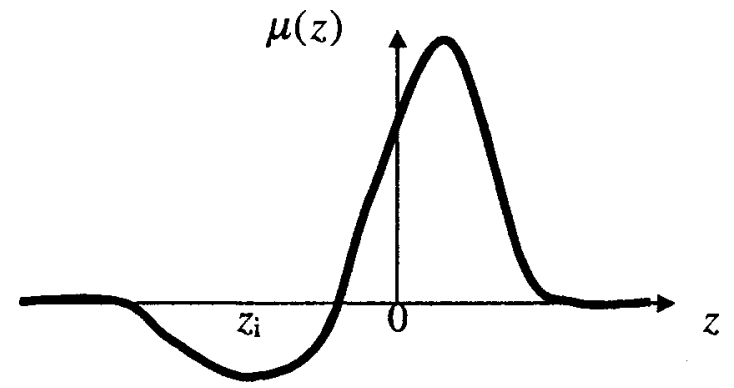

FIG. 6. Schematic presentation of electrochemical potential $\mu(z)$ which has a well and a barrier at the interface.

barrier $V(z, h)$ is different for different $h$ values and so is the transition probability $W_{i \rightarrow f}(h)$. A further scenario depends on the relationship between $W_{i \rightarrow f}(h)$ and diffusion fluxes $j_{h}$ maintaining the equilibrium in the potential well along the interface coordinate $h$. Two limiting cases are feasible.

\section{A. High potential barrier}

When the potential barrier along the $z$ coordinate $V(z, h)$ is high at all $h$ values, even at $h=h_{\max }$, the transition probability $W_{i \rightarrow f}(h)$ is small compared to $j_{h}$. In this case the equilibrium distribution $G_{i}(h)$ over coordinate $h$ is established for the ion residing in the initial potential well of $\mu(z)$. Then the total transition probability is obtained by averaging of $W_{i \rightarrow f}(h)$ over the distribution $G_{i}(h)$ (see Appendix D). The result reads

$$
k_{\text {rate }}=D_{z} \sqrt{\frac{\Omega^{2}}{2 \pi k_{B} T}} \exp \left[-\frac{\mu\left(z_{\max }\right)}{k_{B} T}\right],
$$

where $\mu(z)$ in the neighborhood of the maximum has been approximated as

$$
\mu(z) \approx \mu\left(z_{\max }\right)-\frac{1}{2} \Omega^{2}\left(z-z_{\max }\right)^{2} .
$$

Equation (32) shows that the rate constant is determined by the characteristics of the motion along the ion degree of freedom in spite of the fact that the diffusion coefficient for the ion is much larger than that for the interfacial motion. This is due to a small probability of the escape from the initial potential well. The parameters of the interaction of the ion with the interface are involved here in the shape and the height of the potential barrier. Equation (32) coincides with the result obtained earlier for a static, flat interface between two immiscible liquids. ${ }^{14,21,22}$

\section{B. Moderate potential barrier}

If the potential barrier along the $z$ coordinate $V(z, h)$ is high everywhere except for the values of $h$ close to $h_{\max }$, the transition probability $W_{i \rightarrow f}(h)$ is small compared to $j_{h}$ almost everywhere except for the vicinity of $h=h_{\max }$.

The minimal barrier which allows an easy escape from the initial potential well will be realized at the $h$ value close to $h_{\max }$. The transition probability is determined then by two contributions. The first one is the probability $W_{i \rightarrow f}(h)$ of escaping from the initial potential well along the coordinate $z$ at $h<h_{\max }$, averaged over practically equilibrium distribu- 
tion for the $h$ coordinate. The second one is the diffusion flux along the $h$ coordinate toward the point $h_{\max }$ (see Appendix D). The first contribution in this limit is usually smaller than the second one and the final result for the rate constant has the form (Appendix D)

$$
k_{\text {rate }}=D_{h} \frac{K h_{\max }}{k_{B} T} \exp \left[-\frac{\frac{1}{2} K h_{\max }^{2}}{k_{B} T}\right] .
$$

The ionic current is thus determined by the characteristics of the slow motion of the interfacial degree of freedom. The characteristics of the ion degree of freedom and its interaction with the interface are implicitly involved in the assumption on the $h$ dependence of the transition probability along the ion coordinate: it is small at all $h$ except for the neighborhood of the point $h=h_{\max }$.

Note that this expression is quite different from Eq. (25) obtained in the absence of the potential barrier for $\mu(z)$ : Equation (34) has an explicit activation character, whereas Eq. (25) is purely diffusional. The physical reason of this difference is as follows. In the barrier case considered in this section, fluctuations of the interface affect only the height of the potential barrier, but they have no influence on the position of the precursor potential well along the $z$ coordinate. In the diffusional case the potential well moves continuously in the $z$ direction with the variation of $h$.

Equation (34) coincides, up to the pre-exponential factor, with the result obtained by Marcus. ${ }^{19}$ In the Marcus equation the pre-exponential factor is proportional to the ionic diffusion coefficient while that in Eq. (34) is proportional to $D_{h}$. This difference reflects the two-dimensional character of the coupled motion over $z$ and $h$ coordinates considered in the present work.

\section{DISCUSSION}

We have obtained three laws for the effective diffusion coefficient, subject to different profiles of the equilibrium electrochemical potential of the ion across the interface, $\mu(z)$.

When $\mu(z)$ does not have a barrier near the interface, we get Eq. (30) for any mobility of the ion in the bulk. If it has a barrier preceded by a well (Fig. 6), two solutions are possible, both for the situation when the ion diffusion in the bulk is faster than the relaxation of the interface.

The first of these solutions, Eq. (32), corresponds to the limiting case when the barrier is higher than the effective barrier due to the nonequilibrium character of the interface fluctuation. In this case, the ion transfer is totally determined by the motion over the ion coordinate. The ion transfer along long $z$ coordinate is slowest in this case.

The second solution, Eq. (34), corresponds to the case when the maximum in $\mu(z)$ is lower than the barrier provided by interface fluctuations, and thus the ion has to sit in the well and wait for a favorable fluctuation of the bump height. The exponential in Eq. (34) represents this "gating" effect. In this case, the motion along the coordinate of the interfacial deformation controls the ion transfer.
We now discuss the consequences of these equations, and then summarize how they help us to understand the role of the nature of the liquids and the ions on the ion transfer across the interface.

Equation (26) predicts slowing down of the ion motion relative to its motion in the bulk due to the finite relaxation time of the bump (a kind of "interfacial polaron" effect). Essentially the motion is that much slower as $\left(D_{h} / \beta D_{z}\right)$ is much smaller than 1 , with $\beta \sim\left[h_{\max } / \Lambda\right]^{2}$.

The increase of surface tension decreases $\beta$ and thereby increases $D_{\text {eff }}$. When the polarity of the two liquids differs less, $h_{\max }^{2}$ is smaller and the slowing down is less strong. In general, for $D_{h}$ that is ten times smaller than $D_{z}$, we may expect to get not stronger than ten times slowing down.

The motion of hydrophobic ions may be hampered less than the motion of hydrophilic ions, because for hydrophobic ions $h_{\max }^{2}$ is smaller. Specifically, for the same absolute value of the free energy of ion transfer from one solvent into another one (which is compensated by cell polarization), the slowing down will be less pronounced for a hydrophobic ion. Thus, we may speculate more about weaker slowing down for tetrabutylammonium cation than for $\mathrm{Br}^{-}$, and for tetrapropylammonium cation than for $\mathrm{Cs}^{+}$. Since, furthermore, larger hydrophobic ions have typically smaller bulk mobility, the slowing down may appear to be negligible.

Equation (32) predicts exponentially strong slowing down depending on the barrier in electrochemical potential. For instance, for just the $6 k_{B} T$ barrier the transport will be 500 times slower. Generally, the existence of the barrier is determined by the fine competition between the chemical and electrical parts of the electrochemical potential, ${ }^{14}$ which should be influenced by the Debye length in each of the solvents. For large Debye lengths (low concentration of buffer electrolytes) there should be no barrier due to such competition. However, one can envisage a barrier just in the chemical part of the electrochemical potential. For instance, for hydrophilic ions that tend to form weak aqua complexes with water, it could be hard to strip off their first solvation shells, and that could be apprehended as a "last" obstacle (barrier) for the ion transfer.

Equation (34) suggests that the transport is essentially determined by creating a favorable fluctuation of the bump. The slowing down, suggested by Eq. (34) may be still quite strong, as far as $\gamma h_{\text {max }}^{2} / 2 \gg k_{B} T$. For $h_{\max } \approx 1 \mathrm{~nm}$ and $\gamma$ $\approx 30 \mathrm{dn} / \mathrm{cm}, \gamma h_{\max }^{2} / 2 \approx 4 k_{B} T$. Thus, for $D_{h}$ which is ten times smaller than $D_{z}$, this mechanism results into a 50 times slow down of the ionic transfer. As well as in the barrier free case, the increase of surface tension will weaken the effect, because $h_{\max } \propto 1 / \gamma$. All other tendencies of the barrier-free case will remain in this case as well.

Thus the only case when it is hard to envisage the effect of the nature of the ion or the liquids is the one characterized by a high barrier in $\mu(z)$. The slowing down is essentially determined by the height of the barrier, which results from a number of competing factors. The predictive power of the theory in this case is weak. The existing molecular dynamics simulations do not suggest any remarkable barrier for the chemical potential but they do not tell us anything about the electrochemical potential. For that reason, it would have 
been premature ${ }^{19}$ to bury the barrier theories.

What will be a direct experimental proof of the suggested mechanism of the protrusion assisted ion transfer? According to our model, the ion current should induce a nonequilibrium amplification of the interface fluctuations, which leads to an increase of the interfacial area as compared to the case of zero current. The excess area stored in the interface fluctuations can be measured quantitatively by the micropipet technique. ${ }^{35,36}$ Thus a predicted correlation between the ionic current and the excess area can be verified. Recently, this technique has allowed measuring an amplification of lipid membrane surface, induced by proton pumping. ${ }^{35}$

The self-consistent motion of the ion and the protrusion in front of it, envisaged as a "two-dimensional" trajectory, is something not unusual within the context of multidimensional diffusion kinetics. ${ }^{23}$ However, a natural question could arise: Can ion-induced solvent deformation accelerate the ion motion? For instance, can the ion that moves from oil toward water be sucked by water protrusion (a harpoon effect) into oil with its subsequent motion back to water?

The answer is simple. Such a fluctuation costs energy, and the system in fact chose an optimum trajectory in the plane of protrusion coordinate and ion coordinate, and that is all what the first part of our theory is about. Only if the ion in the bulk were inertialess with respect to the protrusion motions (the "polaron" limit), could it have made sense to speak about harpooning, keeping in mind that the fast ion would be slowed down at the interface, waiting for a proper protrusion fluctuation, but the transport of the ion across the surface can never be faster than in the bulk. Thus harpooning helps to cross the surface by "killing" for a moment the surface barrier, so that when that occurs the ion moves almost like in the bulk, but the ion will have to wait for quite a while for the gate to open.

\section{CONCLUSION}

The ion transfer across a soft interface between two immiscible solutions has been studied. The model involves the consideration of both the ion motion and creation and relaxation of surface protrusion as a result of ion-interface interaction and thermal fluctuations. We have found that three regimes of ion transfer across ITIES can be distinguished.

We have shown that in the absence of the barrier in the electrochemical potential the rate constant for the ionic transfer can be described as a two dimensional diffusion of the coupled system which includes the ion and the interfacial protrusion. Then the rate constant for the transfer across ITIES is determined by the effective diffusion coefficient $D_{\text {eff }}$, for the ion-protrusion couple. The equation for $D_{\text {eff }}$ is derived, which includes the characteristics of the ion and the interface. It is shown that nonequilibrium interface adjustment slows down essentially the ionic transfer. The effect becomes the most pronounced when the relaxation of the interface is much slower than the diffusion of the "bare" ion. Then the kinetics of the ion transfer is controlled by slow relaxation of the interface. We may call this case interfacial polaron limit.
We have found that in the case of the barrier in the electrochemical potential the ionic transfer has an activation character. The effective activation energy is determined by a competition between probabilities to overcome a barrier in $\mu(z)$ and to create a protrusion favorable for the ion transfer. Correspondingly the two limiting behaviors can be distinguished.

For the high barrier in $\mu(z)$ the transfer is completely determined by the slow transition over this barrier, and fluctuations of the interface just follow the ionic motion. Then the derived equation for the rate constant reduces to the one obtained by Gurevich and Kharkats ${ }^{21}$ and by Schmickler. ${ }^{14}$ It should be noted that a similar equation has been derived by Marcus $^{37}$ in the context of a bond breaking/bond formation reaction. We may call this case the "bare" ion hopping.

For a moderate barrier in $\mu(z)$ the rate constant is determined by the time needed for the creation of the favorable protrusion which reduces the barrier for the ionic transition. This mechanism is essentially the same as has been recently proposed by Marcus. ${ }^{19}$ Treatment of "two-dimensional" nature of the motion over the coordinates of the ion and protrusion specifies the pre-exponential factor in the rate constant. We may call this case the gating limit.

The interfacial polaron, bare ion hopping, and gating limits cover essentially all representative cases of the ion transfer across the interface. Through the formulas characterizing these limits, the role of the nature of ion and the liquids in contact on the ion transfer kinetics is more or less straightforwardly revealed. This asks for systematic experimental studies suggested in Discussion.

\section{ACKNOWLEDGMENTS}

The authors thank Takashi Kakiuchi for a useful discussion. The financial support of this work by International Bureau (Research Center "Juelich") and Alexander von Humboldt Foundation, Germany, and by Israel Science Foundation (Grant No. 573\00-2), is greatly appreciated.

\section{APPENDIX A: ELIMINATION OF FAST VARIABLES IN ADIABATIC APPROXIMATION}

The idea of this method $^{20}$ is similar to the BornOppenheimer approximation in quantum mechanics. We expand the distribution function $w(z, h, t)$ in the complete set of the functions $\phi_{n}(Y, X)$ of the fast variable $Y$ considering the slow variable $X$ as a parameter

$$
w(z, h, t)=\sum_{m=0}^{\infty} f_{m}(X, t) \phi_{m}(Y, X) .
$$

In the problem that we study, $Y \equiv z$ for $D_{h} \ll D_{z}$ and $Y \equiv h$ for $D_{h} \ll D_{z}$ while $X \equiv h$ for $D_{h} \ll D_{z}$ and $X \equiv z$ for $D_{h} \ll D_{z}$. The functions $\phi_{n}(Y, X)$ are the eigenfunctions of the operator $\partial J_{Y}(Y, X) / \partial Y$ given by Eqs. (14) or (15)

$$
\rho \frac{\partial}{\partial Y} J_{Y}(Y, X) \phi_{n}(Y, x)=\lambda_{n}(X) \phi_{n}(Y, X) .
$$


Here the slow variable $X$ appears only as a parameter and $\rho=D_{x} / D_{y}$. Taking into account the terms of the zero and the first order in the small parameter $\rho$ we get the following equations for $f_{n}(X, t)$ :

$$
\begin{aligned}
& \frac{\partial f_{0}(X, t)}{\partial t}=\left(J_{0,0}+\rho \sum_{n=1}^{\infty} J_{0, n}\left[\lambda_{n}(X)\right]^{-1} J_{n, 0}\right) f_{0}(X), \\
& f_{n}(X)=\rho\left[\lambda_{n}(X)\right]^{-1} J_{n, 0} f_{0}(X), \quad n \geqslant 1,
\end{aligned}
$$

where the matrix elements $J_{n, m}$ have the form

$$
J_{n, m}=-\int d y \phi_{n}^{+}(Y, X) \frac{\partial}{\partial X} J_{X}(X, Y) \phi_{m}(Y, X),
$$

and $\phi_{n}^{+}(Y, X)$ is the eigenfunction of the operator adjoint to the operator $J_{Y}(Y, X)$. From the orthonormality of the functions $\phi_{n}$ and $\phi_{n}^{+}$it follows that $f_{0}(X, t)$ is the integral of $w(X, Y, t)$ over the fast variable $Y$ (Ref. 20)

$$
f_{0}(X, t)=\int d y w(X, Y, t) .
$$

\section{APPENDIX B: ION COORDINATE AS A SLOW VARIABLE}

For a slow motion of the ion its coordinate $z$ plays a role of the slow variable, $X$, while the height of the protrusion $h$ represents the fast variable $Y$. Then the eigenvalues $\lambda_{n}(z)$ and the eigenfunctions $\phi_{n}$ and $\phi_{n}^{+}$defined by Eq. (A2) read

$$
\begin{aligned}
\lambda_{n}(z)= & n K / \eta_{z}, \\
\phi_{n}(h, z)= & \left(\frac{K}{2 \pi k_{B} T}\right)^{1 / 2} \frac{1}{2^{n} n !} H_{n}\left(\left(\frac{K}{2 k_{B} T}\right)^{1 / 2}\left(h-h_{\mathrm{eq}}(z)\right)\right) \\
& \times \exp \left(\left(K / 2 k_{B} T\right)\left(h-h_{\mathrm{eq}}(z)\right)^{2}\right), \\
\phi_{n}^{+}(h, z)= & H_{n}\left(\left(K / 2 k_{B} T\right)^{1 / 2}\left(h-h_{\mathrm{eq}}(z)\right)\right),
\end{aligned}
$$

where $H_{n}$ are Hermit polynomials. ${ }^{38}$ Substituting Eqs. (B2) and (B3) into Eq. (A5) we find

$$
\begin{aligned}
J_{0,1}= & {\left[\frac{\partial}{\partial z}\left(\frac{d \mu(z)}{d z}\right)+\frac{k_{B} T}{\gamma_{z}} \frac{\partial^{2}}{\partial z^{2}}\right] \delta_{0, n} } \\
& -\left(\frac{k_{B} T}{2 K}\right)^{1 / 2} \frac{K}{\gamma_{z}} \frac{\partial}{\partial z} \frac{d h_{\mathrm{eq}}(z)}{d z} \delta_{1, n} .
\end{aligned}
$$

In order to find matrix elements of $f_{0}(z)$ [entering Eq. (A3)], due to the presence of the Kronecker symbols $\delta_{0, n}$ and $\delta_{1, n}$ in Eq. (B4), we need to calculate $J_{1,0}$ only. The latter reads as

$$
\begin{aligned}
J_{1,0}= & \left(2 k_{B} T K\right)^{1 / 2} \frac{1}{\eta_{z}}\left[-\frac{\partial}{\partial z}+\frac{\eta_{z}}{k_{B} T} \frac{d h_{\mathrm{eq}}(z)}{d z} \frac{d \mu(z)}{d z}\right. \\
& \left.+2 \frac{d h_{\mathrm{eq}}(z)}{d z} \frac{\partial}{\partial z}+\frac{d^{2} h_{\mathrm{eq}}(z)}{d z^{2}}\right] .
\end{aligned}
$$

A comparison of Eqs. (16) and (A6) shows that in the case of slow ionic motion the ionic concentration $c(z)$ is given by a function $f_{0}(z)$ which had been introduced in Appendix A. Then substituting Eqs. (B4) and (B5) into Eq. (A3) we arrive at Eq. (19) in the text.

\section{APPENDIX C: HEIGHT OF THE PROTRUSION AS A SLOW VARIABLE}

In this case the ionic coordinate $z$ plays a role of the fast variable $Y$. The slowness of motion along the $h$ coordinate results in the Boltzmann equilibrium over $z$ being established at every value of $h$. The effective force acting on the interface $\partial V_{\text {eff }}(h) / \partial h$ is obtained after averaging $\partial V(z, h) / \partial h$ over $z$. At a given $h$, the result is determined by the behavior of $V(z, h=$ const. $)$ in the vicinity of its minima over $z$.

According to Eq. (11) the potential $V(z, h=$ const.) has minima at points $z_{j}(h)$ which are the solutions of the equation $h=h_{\mathrm{eq}}(z)$. In our model [see Figs. 1(b) and 2(b)] there are at least two minima, one lying at $z<h_{\max }$ and another lying at $z>h_{\max }$, where $h_{\max }$ is the maximum height of the protrusion. By definition the maximum of the function $h_{\mathrm{eq}}(z)$ lies at $z=h_{\max }$. Below we calculate the distribution function $w(z, h, t)$ in the intervals $z<h_{\max }$ and $z>h_{\max }$ separately.

If in a fairly wide vicinity of the points $z_{j}(h)$ the quadratic expansion of the potential $V(z, h)$ is correct then the potential $V(z, h)$ in the Fokker-Planck Eq. (13) can be approximated by

$$
V(z, h)=\mu\left(z_{j}(h)\right)+\frac{K}{2} \alpha_{j}^{2}(h)\left(z-z_{j}(h)\right)^{2}
$$

in the regions $z<h_{\max }$ and $z>h_{\max }$, respectively, where $\alpha_{j}(h)=d h_{\mathrm{eq}}(z) /\left.d z\right|_{z=z_{j}(h)}$. Then in the corresponding regions the eigenvalues $\lambda_{n}(z)$ and the eigenfunctions $\phi_{n}$ and $\phi_{n}^{+}$defined by Eqs. (A2) have the form

$$
\begin{aligned}
\lambda_{n}(h)= & \frac{n K}{\eta_{h}} \alpha_{j}^{2}(h), \\
\phi_{n}(z, k)= & \left(\frac{K \alpha_{j}^{2}(h)}{2 \pi k_{B} T}\right)^{1 / 2} \frac{1}{2^{n} n !} H_{n}\left(\left(\frac{K \alpha_{j}^{2}(h)}{2 k_{B} T}\right)^{1 / 2}\left(z-z_{j}(h)\right)\right) \\
& \times \exp \left[-\frac{K \alpha_{j}^{2}(h)}{2 k_{B} T}\left(z-z_{j}(h)\right)^{2}\right],
\end{aligned}
$$

$\phi_{n}^{+}(z, h)=H_{n}\left(\left(\frac{K \alpha_{j}^{2}(h)}{2 k_{B} T}\right)^{1 / 2}\left(z-z_{j}(h)\right)\right)$.

Here for simplicity we presented results for the case of zero driving force $\mu(z)=0$. The same approach can also be used for a linear profile of $\mu(z)$.

Substituting Eqs. (C3) and (C4) into Eqs. (A2)-(A4) we obtain the following expressions for the steady-state distributions $f_{0}(h)$ and $f_{1}(h)$ :

$$
f_{0}(h)=A_{j} h+B_{j}, \quad f_{1}(h)=A_{j} \frac{D_{h}}{D_{z} \alpha_{j}(h)}, \quad j=1,2 .
$$

In writing Eq. (C5) we kept only the leading terms in the small parameter $D_{h} / D_{z}$. The coefficients $A_{1,2}$ and $B_{1,2}$ entering Eq. (C5) should be found from the boundary conditions Eq. (18) and from continuity of the ionic concentration and of the flux at $z=h_{\max }$.

It should be noted that Eqs. (C2)-(C5) are defined only for the values of $h$ for which the equation $h=h_{\text {eq }}(z)$ has a solution. We limit our consideration by this range of heights 
of the protrusions because they give the major contribution to the ionic current averaged over the distribution of $h$.

For the triangle shape of the function $h_{\mathrm{eq}}(z), j=1,2$

$$
z_{j}(h)=h_{\max }+(-1)^{j}\left(h_{\max }-h\right) \Lambda / h_{\max },
$$

and

$$
\alpha_{j}(h)=(-1)^{j+1} h_{\max } / \Lambda .
$$

Applying Eq. (A1) and Eqs. (C3)-(C5) one comes finally to Eq. (25) for the rate constant.

\section{APPENDIX D: KINETICS IN THE PRESENCE OF A BARRIER IN $\mu(z)$}

\section{General equations}

Assuming that the motions of the interface are so slow that equilibrium with respect to an ion motion along the $z$ axis has time to establish. Thus as a first approximation we may put $J_{z} w(z, h, t)=0$ where the operator $J_{z}$ is defined by Eq. (14). Hence in the initial well the steady-state distribution function $w(z, h)$ has the form

$$
\begin{aligned}
w(z, h) & =G_{i}(h) Z^{-1}(h) \exp \left[-\frac{\mu(z)+\frac{1}{2} K\left[h-h_{\max }(z)\right]^{2}}{k_{B} T}\right] \\
& =G_{i}(h) Z^{-1}(h) \exp \left[-\frac{V(z, h)}{k_{B} T}\right],
\end{aligned}
$$

where

$$
Z(h)=\int_{(i)} d z \exp \left\{-\frac{\mu(z)+\frac{1}{2} K\left[h-h_{\max }(z)\right]^{2}}{k_{B} T}\right\},
$$

with the integration in Eq. (D2) performed over the initial well. The function $G_{i}(h)$ should be found solving Eq. (13). $\partial w / \partial t$ in Eq. (13) now cannot be ignored. On the contrary, our task will be to study the process of exhaustion of the population in the initial well in time.

Inserting Eq. (D1) into Eq. (13) and integrating over $z$ we obtain

$$
\begin{aligned}
\frac{\partial G_{i}}{\partial t}= & j_{z}(-\infty)-j_{z}\left(z_{\max }(h)\right)-D_{h} \frac{\partial}{\partial h}\left\{\frac{\partial G_{i}}{\partial h}\right. \\
& \left.+\frac{G_{i}(h)}{k_{B} T} \int_{(i)} d z Z^{-1}(h) \exp \left[-\frac{V(z, h)}{k_{B} T}\right] \frac{\partial V(z, h)}{\partial h}\right\},
\end{aligned}
$$

where $j_{z}$ is the flux along $z$ coordinate and $z_{\max }(h)$ is the coordinate of the barrier maximum.

Before the concentration of ions in the well is exhausted the flux $j_{z}(-\infty)$ may be neglected; putting $j_{z}(-\infty)=0$ is a standard approximation in the calculation of the escape probabilities. ${ }^{39}$ The flux at the top of the barrier is equal to

$$
j_{z}\left(z_{\max }(h)\right)=W_{i \rightarrow f}(h) G_{i}(h),
$$

where $W_{i \rightarrow f}(h)$ is the transition probability per unit time for the escape from the initial potential well at a given $h$

$$
W_{i \rightarrow f}(h)=\frac{D_{z}}{\int_{z_{0_{i}}}^{z_{0_{f}}} d z \exp \left\{\frac{\mu(z)+\frac{1}{2} K\left[h-h_{\mathrm{eq}}(z)\right]^{2}}{k_{B} T}\right\} \int_{i} d z \exp \left\{-\frac{\mu(z)+\frac{1}{2} K\left[h-h_{\mathrm{eq}}(z)\right]^{2}}{k_{B} T}\right\}} .
$$

Let us now rewrite Eq. (D3) in the form

$$
\frac{\partial G_{i}}{\partial t}=D_{h} \frac{\partial}{\partial h}\left\{\frac{\partial G_{i}}{\partial h}+\frac{1}{k_{B} T} \frac{\partial U_{\mathrm{eff}}(h)}{\partial h} G_{i}\right\}-W_{i \rightarrow f}(h) G_{i} .
$$

Here we have introduced an effective potential

$$
U_{\mathrm{eff}}(h)=-k_{B} T \ln \left[\frac{\int_{i} d z \exp \left\{-\frac{\mu(z)+\frac{1}{2} K\left[h-h_{\mathrm{eq}}(z)\right]^{2}}{k_{B} T}\right\}}{\int_{i} d z \exp \left\{-\frac{\mu(z)}{k_{B} T}\right\}}\right],
$$

which depends strongly on the shape of $\mu(z)$. If $\mu(z)$ behaves as shown in Fig. 6, the effective potential for $h$ is close to the parabolic form. If on the contrary $\mu(z)$ is constant, the effective potential in Eq. (D7) does not depend on $h$. In the latter case we return to the situation considered in Sec. V.

Equation (D6) is a nonlinear integro-differential equation which is hard to solve. We consider two particular cases subject to two limiting values of the ratio of the local transition probability [Eq. (D5)] and the diffusion fluxes

$$
j_{h}=\frac{D_{h}}{\left(k_{B} T\right)^{2}}\left[\frac{\partial U_{\mathrm{eff}}}{\partial h}\right]^{2} .
$$

Case 1: $W_{i \rightarrow f}(h)$ is small compared to $j_{h}$ at all $h$ values

Here we can omit the last term on the right-hand side of Eq. (D6). The rest of the equation can be easily integrated to obtain

$$
G_{i}(h)=\frac{\exp \left\{-\frac{U_{\mathrm{eff}}(h)}{k_{B} T}\right\}}{\int_{i} \exp \left\{-\frac{U_{\mathrm{eff}}(h)}{k_{B} T}\right\} d h} .
$$

This gives for the total transition probability per unit time (i.e., for the average flux) 


$$
\left\langle j_{z}\right\rangle=\int d h \frac{\exp \left\{-\frac{U_{\text {eff }}(h)}{k_{B} T}\right\}}{\int_{i} \exp \left\{-\frac{U_{\text {eff }}(h)}{k_{B} T}\right\} d h} W_{i \rightarrow f}(h) .
$$

Inserting the definitions of $U_{\text {eff }}$ and $W_{i \rightarrow f}$ into Eq. (D10) we obtain approximately

$$
\left\langle j_{z}\right\rangle=D_{z} \frac{\int d h \sqrt{\frac{\left|V_{z z}^{\prime \prime}\left(z_{\max }(h)\right)\right|}{2 \pi k_{B} T}} \exp \left\{-\frac{V\left(z_{\max }, h\right)}{k_{B} T}\right\}}{\int_{i} \exp \left\{-\frac{U_{\mathrm{eff}}(h)}{k_{B} T}\right\} d h \times \int_{i} d z \exp -\frac{\mu(z)}{k_{B} T}},
$$

where an approximate expression for $V(z, h)$ was used

$$
V(z, h) \approx V\left(z_{\max }, h\right)+\frac{1}{2} V_{z z}^{\prime \prime}\left(z_{\max }, h\right)\left(z-z_{\max }\right)^{2}
$$

with the equation on $z_{\max }$

$$
\partial \mu / \partial z-K\left[h-h_{\mathrm{eq}}(z)\right] \partial h_{\mathrm{eq}}(z) / \partial z=0 .
$$

Using approximation (33) we obtain from Eq. (D11)

$$
\left\langle j_{z}\right\rangle=\frac{D_{z}}{2 \pi k_{B} T} \Omega \omega_{\mu} \exp \left[-\frac{\mu\left(z_{\max }\right)}{k_{B} T}\right],
$$

where $\omega_{\mu}$ is the "curvature" of $\mu(z)$ at the bottom of the initial potential well.

This flux describes the probability (per unit time) for the particle to leave the initial potential well and cross the interface. The velocity is determined by the product of the transition probability and the size of the region $L_{i}$ (which the particle has to pass crossing the interface). This gives the rate constant

$$
k_{\text {rate }}=L_{i}\left\langle j_{z}\right\rangle .
$$

The length $L_{i}$ is determined by the normalization integral and is equal to

$$
L_{i}=\sqrt{\frac{2 \pi k_{B} T}{\omega_{\mu}^{2}}} .
$$

Thus we obtain for the rate constant Eq. (32) in the text.

Case 2: $W_{i \rightarrow f}(h)$ is small compared to $j_{h}$ at all $h$ values, except a neighborhood of a point $h_{\max }$.

The general expression for transition probability in this case can be written as ${ }^{39,40}$

$$
j_{z}=\frac{\int_{-\infty}^{z_{\max }} d h W_{i \rightarrow f}(h) \exp \left\{-\frac{U_{\text {eff }}(h)}{k_{B} T}\right\}}{\int_{i} \exp \left\{-\frac{U_{\text {eff }}(h)}{k_{B} T}\right\} d h}+j_{\text {diff }}\left(z_{\max }\right),
$$

where

$$
\begin{aligned}
j_{\text {diff }}\left(z_{0}\right)= & D_{h} \frac{U_{\text {eff }}^{\prime \prime}\left(z_{\text {max }}\right)}{k_{B} T} \sqrt{\frac{U_{\text {eff }}\left(z_{\text {max }}\right)-U_{\text {eff }}\left(h_{i}\right)}{\pi k_{B} T}} \\
& \times \exp \left\{\frac{U_{\text {eff }}\left(z_{\text {max }}\right)-U_{\text {eff }}\left(h_{i}\right)}{k_{B} T}\right\} .
\end{aligned}
$$

The first term in Eq. (D17) is approximately half of the term given by Eq. (D10). Using the definition of $U_{\text {eff }}$ we can write

$$
\begin{aligned}
\exp \left[-\frac{U_{\text {eff }}\left(z_{\text {max }}\right)}{k_{B} T}\right] & \int_{i} d z \exp \left\{-\frac{\mu(z)+\frac{1}{2} K\left[z_{\max }-h_{\mathrm{eq}}(z)\right]^{2}}{k_{B} T}\right\} \\
= & \int_{i} d z \exp \left\{-\frac{\mu(z)}{k_{B} T}\right\} \\
& \approx \exp \left[-\frac{\frac{1}{2} K z_{\max }^{2}}{k_{B} T}\right] .
\end{aligned}
$$

In a similar way we obtain $U_{\text {eff }}\left(h_{i}\right)=0$ substituting 0 for $z_{\max }$ in Eq. (D19). Taking into account that $U_{z z}^{\prime \prime}\left(z_{\max }\right) \approx K$ we obtain

$$
j_{\text {diff }}=D_{h} \frac{K}{k_{B} T} \sqrt{\frac{K z_{\max }^{2}}{2 \pi k_{B} T}} \exp \left[-\frac{\frac{1}{2} K z_{\max }^{2}}{k_{B} T}\right] .
$$

The length $L_{i}$ in this case is equal to

$$
L_{i}=\sqrt{\frac{2 \pi k_{B} T}{K}} .
$$

Taking into account that $z_{\max }=h_{\max }$ we finally arrive at Eq. (34) in the text.

${ }^{1}$ Soft Matter Physics, edited by M. Daoud and C. E. Williams (Springer, Berlin, 1999).

${ }^{2}$ L. I. Daikhin, A. A. Kornyshev, and M. Urbakh, J. Electroanal. Chem. 483, 68 (2000).

${ }^{3}$ C. M. Starks, C. L. Liotia, and M. Halpern, Phase Transfer Catalysis (Chapman and Hall, New York, 1994).

${ }^{4}$ H. H. Girault and D. H. Schiffrin, in Electroanalytical Chemistry, edited by A. J. Bard (Marcel Dekker, New York, 1989), Vol. 15, p. 1; H. H. Girault, in Modern Aspects of Electrochemistry, edited by J. O'M Bockris et al. (Plenum, New York, 1993), Vol. 25, p. 1.

${ }^{5}$ Z. Samec and T. Kakiuchi, in Advanced Electrochemical Electrochem Science, edited by E. Gersicher and C. W. Tobias (VCH, Weinheim, 1995), p. 297.

${ }^{6}$ C. M. Pereira, W. Schmickler, F. Silva, and M. J. Sousa, Chem. Phys. Lett. 268, 13 (1997).

${ }^{7}$ L. I. Daikhin, A. A. Kornyshev, and M. Urbakh, J. Electroanal. Chem. 500, 461 (2001).

${ }^{8}$ C. Gavach, B. d'Epenoux, and F. Henry, J. Electroanal. Chem. 64, 107 (1975); B. d'Epenoux, P. Seta, G. Amblard, and C. Gavach, ibid. 99, 77 (1979); Z. Samec and V. Marecek, ibid. 200, 17 (1986); T. Wandlowski, V. Marecek, K. Holub, and Z. Samec, J. Phys. Chem. 93, 8204 (1989); T. Kakiuchi, J. Noguchi, M. Kotani, and M. Senda, J. Electroanal. Chem. 296, 517 (1990); T. Wandlowski, V. Marecek, Z. Samec, and R. Fuoco, ibid. 331, 765 (1992); T. Kakiuchi, J. Noguchi, and M. Senda, ibid. 336, 137 (1992); V. Marecek, A. Lhotsky, and S. Racinsky, Electrochim. Acta 40, 2909 (1995); P. D. Beattie, A. Delay, and H. H. Girault, ibid. 40, 2961 (1995); T. Kakiuchi and Y. Teranishi, J. Electroanal. Chem. 396, 401 (1995); A. Lhotsky, K. Holub, P. Neuzil, and V. Marecek, J. Chem. Soc., Faraday Trans. 92, 3851 (1996); N. Nishi, K. Izawa, M. Yamamoto, and T. Kakiuchi, J. Phys. Chem. B 105, 8162 (2001).

${ }^{9}$ C. Gavach, J. Chim. Phys. Phys.-Chim. Biol. 70, 1478 (1973); Z. Samec and V. Marecek, J. Electroanal. Chem. 100, 841 (1979); T. Osakai, T. Kakutani, and M. Senda, Bull. Chem. Soc. Jpn. 57, 370 (1984); H. H. Girault and D. J. Schiffrin, J. Electroanal. Chem. 195, 213 (1985).

${ }^{10}$ Y. Shao and H. H. Girault, J. Electroanal. Chem. 282, 59 (1990).

${ }^{11}$ Y. Shao, J. A. Campbell, and H. H. Girault, J. Electroanal. Chem. 300, 415 (1991)

${ }^{12}$ T. Kakiuchi, J. Electroanal. Chem. 322, 55 (1992). 
${ }^{13}$ D. E. Goldman, J. Gen. Physiol. 27, 37 (1943).

${ }^{14}$ W. Schmickler, J. Electroanal. Chem. 426, 5 (1997).

${ }^{15}$ K. Aoki, Electrochim. Acta 41, 2321 (1996).

${ }^{16}$ A. V. Indenbom, Electrochim. Acta 40, 2985 (1995).

${ }^{17}$ I. Benjamin, J. Chem. Phys. 97, 1432 (1992); Science 261, 1558 (1993).

${ }^{18}$ K. J. Schweighofer and I. Benjamin, J. Phys. Chem. A 103, 10274 (1999).

${ }^{19}$ R. A. Marcus, J. Chem. Phys. 113, 1618 (2000).

${ }^{20} \mathrm{H}$. Risken, The Fokker-Planck Equation (Springer, Berlin, 1996).

${ }^{21}$ Yu. Ya. Gurevich and Yu. I. Kharkats, Sov. Electrochem. 22, 463 (1986).

${ }^{22}$ A. M. Kuznetsov and Yu. I. Kharkats, in The Interface Structure and Electrochemical Processes at the Boundary between Two Immiscible Liquids, edited by V. E. Kazarinov (Springer, Berlin, 1987), p. 11.

${ }^{23}$ A. M. Kuznetsov, Charge Transfer in Physics, Chemistry and Biology (Gordon and Breach, Reading, 1995).

${ }^{24}$ O. Pecina, W. Schmickler, and E. Spohr, J. Electroanal. Chem. 394, 29 (1995).

${ }^{25}$ M. T. M. Koper and W. Schmickler, Chem. Phys. 211, 123 (1996).

${ }^{26}$ L. D. Landau and E. M. Lifshitz, Electrodynamics of Continuous Media, Theoretical Physics, Vol. 8 (Pergamon, New York, 1984).

${ }^{27}$ E. Rafael and P.-G. de Gennes, Europhys. Lett. 31, 293 (1995).

${ }^{28}$ R. J. Phillips and S. R. Dungan, J. Colloid Interface Sci. 201, 48 (1998).

${ }^{29}$ A. A. Kornyshev, in The Chemical Physics of Solvation, edited by R. R.
Dogonadze, E. Kalman, A. A. Kornyshev, and J. Ulstrup (Elsevier, Amsterdam, 1985), part A, p. 77.

${ }^{30}$ M. A. Vorotyntsev and A. A. Kornyshev, Electrostatics of Media with the Spatial Dispersion (Nauka, Moscow, 1993).

${ }^{31}$ C. A. Croxton, Statistical Mechanics of the Liquid Surface (Wiley, New York, 1980).

${ }^{32}$ J. S. Rowlinson and B. Widom, Molecular Theory of Capillarity (Clarendon, Oxford, 1982).

${ }^{33}$ R. Ferrigno and H. H. Girault, J. Electroanal. Chem. 496, 131 (2001).

${ }^{34}$ Strictly speaking $c\left(z_{f}\right) \neq 0$ and one should have included also the backward reaction, which we neglect here for simplicity. This could be done along the line described below.

${ }^{35}$ J.-B. Manneville, P. Bassereau, D. Levy, and J. Prost, Phys. Rev. Lett. 82, 4356 (1999)

${ }^{36}$ R. M. Allen, K. Konturri, L. Murtomaki, and D. E. Williams, J. Electroanal. Chem. 483, 57 (2000).

${ }^{37}$ R. A. Marcus, J. Phys. Chem. 72, 891 (1968).

${ }^{38}$ I. S. Gradshtein and I. M. Ryzhik, Table of Integrals, Series, and Products (Academic, New York, 1980)

${ }^{39}$ A. M. Kuznetsov, Stochastic and Dynamic Views of Chemical Reaction Kinetics in Solutions (Presses Polytechniques, Lausanne, 1999).

${ }^{40}$ A. M. Berezhkovskii and V. Yu. Zitzerman, Chem. Phys. 164, 341 (1992). 